\title{
Quantum phase transition in the SU(4) spin-orbital model on the triangular lattice
}

\author{
Karlo Penc, ${ }^{1,2}$ Matthieu Mambrini, ${ }^{3}$ Patrik Fazekas, ${ }^{2}$ and Frédéric Mila ${ }^{4}$ \\ ${ }^{1}$ Max-Planck-Insitut für Physik Komplexer Systeme, Nöthnitzer Strasse 38, 01187 Dresden, Germany \\ ${ }^{2}$ Research Institute for Solid State Physics and Optics, H-1525 Budapest, P.O.B. 49, Budapest, Hungary \\ ${ }^{3}$ Laboratoire de Physique Quantique, Université Paul Sabatier, 31062 Toulouse Cedex 04, France \\ ${ }^{4}$ Institut de Physique Théorique, Université de Lausanne, CH-1015 Lausanne, Switzerland
}

(Received 7 May 2003; published 21 July 2003)

\begin{abstract}
Motivated by the absence of a cooperative Jahn-Teller effect in $\mathrm{LiNiO}_{2}$ and $\mathrm{BaVS}_{3}$, two layered oxides with triangular planes, we study the SU(4) symmetric spin-orbital model on the triangular lattice. Upon reducing the next-nearest-neighbor coupling, we show that the system undergoes a quantum phase transition to a liquid phase. A variational approach to this liquid phase shows that simple types of long-range correlations are suppressed, suggesting that it is stable against lattice distortions.
\end{abstract}

DOI: 10.1103/PhysRevB.68.012408

The possibility to enhance quantum fluctuations and destroy magnetic long-range order by orbital fluctuations was proposed a few years ago in the pioneering work of Feiner $e t$ al. ${ }^{1}$ A careful analysis of the spin-orbital model of cubic perovskites with twofold orbital degeneracy revealed the presence of spin liquid phases in which orbital degrees of freedom form valence-bond patterns. The search for experimental realizations of this intriguing physics has been going on since then, but the best candidate remains the old system $\mathrm{LiNiO}_{2},{ }^{2}$ a layered $S=1 / 2$ system which does not undergo any phase transition down to the lowest temperatures. In this system, $\mathrm{Ni}^{3+}$ ions are arranged in triangular layers, and since the $\mathrm{SU}(2)$ Heisenberg model on a triangular lattice is now believed to sustain long-range order, ${ }^{3}$ the origin of this behavior has logically been attributed to the twofold orbital degeneracy of $\mathrm{Ni}^{3+}$ in the low-spin state. However, the absence of any structural distortions, which one would expect, e.g., from a valence-bond-ordered spin liquid of Ref. 1, is still a mystery. Similar physics seems to be present in $\mathrm{BaVS}_{3},{ }^{4}$ a $3 d$ (Ref. 1) system with stacked triangular planes: Between $30 \mathrm{~K}$ and $70 \mathrm{~K}$ it develops a spin gapped phase, again without significant lattice distortions. We believe that essential aspects of this intermediate phase are also describable in terms of a triangular spin-orbital model with twofold orbital degeneracy.

On the theory side, it has become clear since the work of Feiner et al. ${ }^{1}$ that states in which orbitals form valence-bond dimer patterns do not exhaust the possibilities of spin liquid states. In particular, increasing attention has been devoted to the SU(4) symmetric Kugel-Khomskii model, ${ }^{5}$ where the orbital and spin degrees of freedom play symmetric roles. In terms of spin-1/2 operators $\vec{\sigma}_{i}$ for the spins and pseudospin$1 / 2$ operators $\vec{\tau}_{i}$ for the orbitals, this model is defined by the Hamiltonian

$$
\mathcal{H}=\sum_{(i, j)} J_{i j}\left(2 \vec{\sigma}_{i} \cdot \vec{\sigma}_{j}+\frac{1}{2}\right)\left(2 \vec{\tau}_{i} \cdot \vec{\tau}_{j}+\frac{1}{2}\right) .
$$

This Hamiltonian can be rewritten as $\mathcal{H}=\Sigma_{(i, j)} J_{i j} P_{i, j}$, where $P_{i, j}$ permutes the states of the four-dimensional fundamental representations of $\mathrm{SU}(4)$ at sites $i$ and $j$. In one dimension, this model has a gapless spectrum with a fourfold periodicity of correlation functions. ${ }^{6}$ The investigation of this
PACS number(s): 75.10.Jm, 75.40.Cx, 75.40.Gb

model on other simple lattices has revealed that, whenever possible, there is a tendency to form four-site plaquettes to accommodate $\mathrm{SU}(4)$ singlets, ${ }^{7}$ in contrast to the orbital valence-bond solids. These plaquettes have been unambiguously shown to form a well-defined pattern in the case of ladders ${ }^{8}$ and evidence that this might also be the case for the square lattice has been put forward. ${ }^{9,10}$ Alternatively, longrange order (LRO) has been proposed in Ref. 11. It is interesting to note that this $\mathrm{SU}(4)$ model is also a good starting point to describe $\mathrm{CeB}_{6}$ (e.g., see Ref. 12), while the other version with conjugate representations on different sublattices is more appropriate in the context of strong coupling to the lattice, ${ }^{13}$ or to perform a $1 / N$ expansion. ${ }^{14}$

In this paper, we present strong evidence, all based on arguments that respect the underlying SU(4) symmetry, that the SU(4) model on a triangular lattice with nearest-neighbor (NN) exchange $J>0$ only is also a spin liquid, but unlike previous suggestions, ${ }^{7,10}$ we put forward detailed variational arguments that go against the presence of plaquette order in the ground state. Extending the model by including a coupling $J^{\prime}>0$ between next-nearest neighbors (NNNs), we prove that the model undergoes a quantum phase transition between a disordered state for $J^{\prime}=0$ and an ordered state for large enough $J^{\prime}$. The emerging picture of the disordered phase is that of a true liquid both in spin and orbital sectors, with accordingly no tendency to undergo any distortion when coupled to the lattice.

The infinite degeneracy of the antiferromagnetic, fourstate Potts model on the triangular lattice is lifted by $J^{\prime}$ $>0$, leading to a unique, four-sublattice ordering pattern [see Fig. 1(a)]. Once the classical state is ordered, quantum fluctuations can be analyzed within a flavor-wave theory, ${ }^{15}$ which is a generalization of the standard SU(2) linearized spin-wave theory. First, one extends the fundamental representation on a single site into a Young diagram with a single row and $M$ columns (the fully symmetric representation). The SU(4) operators can be conveniently expressed by a generalized Holstein-Primakoff transformation, where the fluctuations on each site are described by three different bosons $b_{n}^{m}(l)$ with $m \neq n$, for which the vacuum state is the $M$-fold direct product of the state $|m\rangle$ associated with the particular sublattice [Fig. 1(a)]. Following the standard procedure, ${ }^{15}$ i.e., expanding in $1 / M$ and keeping the qua- 

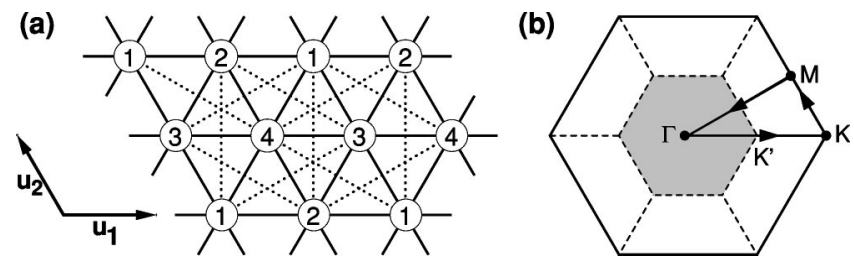

FIG. 1. (a) Four-sublattice long-range-ordered state with basis vectors $\mathbf{u}_{1}=(1,0)$ and $\mathbf{u}_{2}=(-1 / 2, \sqrt{3} / 2)$. The dashed line represents the next-nearest-neighbor exchange. The sublattices are labeled according to the occupation by the states of the fundamental representation. (b) Brillouin zone of the triangular lattice. The smaller, shaded hexagon denotes the reduced Brillouin zone associated with the four-sublattice order.

dratic bosonic terms, the Hamiltonian can be diagonalized and, up to a constant, is given by

$$
\mathcal{H}_{\mathrm{FW}}=\sum_{\mathbf{k} \in \mathrm{RBZ}} \sum_{m, n} \omega_{m n}(\mathbf{k})\left[\alpha_{m, \mathbf{k}}^{n \dagger} \alpha_{m, \mathbf{k}}^{n}+\frac{1}{2}\right]
$$

The energies of the flavor modes are given by

$$
\omega_{m n}(k)=2 \sqrt{\left(J+J^{\prime}\right)^{2}-\left[J \cos \left(\mathbf{k r}_{m n}\right)+J^{\prime} \cos \left(\mathbf{k} \mathbf{r}_{m n}^{\prime}\right)\right]^{2}},
$$

where $\mathbf{r}_{m n}$ and $\mathbf{r}_{m n}^{\prime}$ are the NN and NNN distances between sublattices $m$ and $n$. There are 12 branches in the reduced Brillouin zone [RBZ, Fig. 1(b)], which is equivalent to three in the normal Brillouin zone. The operators $\alpha$ and $b$ are related through a Bogoliubov transformation.

In Fig. 2 we show a plot of the flavor dispersion for some arbitrary value of $J^{\prime} / J$. For $J^{\prime}=0$, the energy of the flavor excitations becomes effectively one dimensional ${ }^{15}$ [e.g., $\omega_{12}(\mathbf{k})=2 J\left|\sin k_{x}\right|$, with a zero energy node along the $k_{x}$ $=0$ line). These low-energy quantum fluctuations destroy the four-sublattice long-range order. Including $J^{\prime}$, the magnons along the $\Gamma-M$ line acquire finite dispersion $\propto \sqrt{J J^{\prime}}$, and this will reduce the fluctuations and stabilize the LRO state.

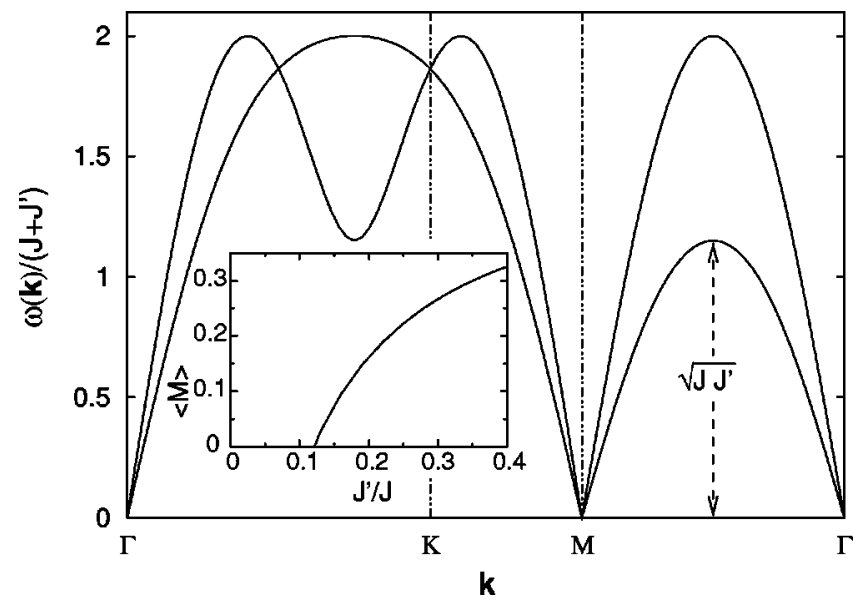

FIG. 2. Flavor-wave dispersion for $J^{\prime} / J=0.1$. Inset: Reduced moment as a function of $J^{\prime} / J$.
Quantitatively the fluctuations are reflected in the effective staggered moment $\langle M\rangle=M-\left\langle\sum_{i \neq m} b_{i}^{m \dagger}(l) b_{i}^{m}(l)\right\rangle$, which reads

$$
\begin{aligned}
\langle M\rangle & =M-\sum_{n \neq m}\left\langle\frac{J+J^{\prime}}{\omega_{m n}(\mathbf{k})}-\frac{1}{2}\right\rangle_{\mathrm{RBZ}} \\
& \approx M+\frac{3}{2}+\frac{3}{2 \pi} \ln \frac{J^{\prime}}{16 J}+\mathcal{O}\left(J^{\prime} / J\right) .
\end{aligned}
$$

The logarithmic divergence is the manifestation of the lowenergy modes. The $\langle M\rangle$ for $M=1$ is finite when $J^{\prime} / J$ $\geq 0.12$ (inset in Fig. 2), and we take this criterion for the stability of the LRO. Note that, within the linear flavor-wave theory, the staggered moments and the energy are symmetric in $J$ and $J^{\prime}$. So we expect LRO to be stable for $0.12 \leq J^{\prime} / J$ $\lesssim 8$ (for $J=0$ we have four decoupled intercalated triangular lattices).

To assess the validity of these results in the extreme quantum limit of the fundamental representation, we have looked for signatures of the quantum phase transition in the exact spectrum on a finite system. Specifically, we have numerically diagonalized a 12 -site cluster defined by the lattice vectors $\mathbf{T}_{1}=2 \mathbf{u}_{1}+4 \mathbf{u}_{2}$ and $\mathbf{T}_{2}=4 \mathbf{u}_{1}+2 \mathbf{u}_{2}$. This particular cluster inherits the $D_{6}$ symmetry of the triangular lattice, and it is also compatible with the four-sublattice ordering. Our aim was to identify the existence of the so-called Anderson tower spectrum, a set of states well separated from the others, which become degenerate and give rise to a linear combination with LRO in the thermodynamic limit. ${ }^{3,16}$ For SU(2) models, their energy for an $N$-site system, measured from the ground state, is proportional to $\mathbf{S}^{2} / N$, where the total spin $S$ is the addition of sublattice spins which maximize the sublattice magnetizations. ${ }^{17}$ In the SU(4) case, the same formula is expected to hold if $\mathbf{S}^{2}$ is replaced by the first Casimir operator $C$ of the SU(4) algebra (see Ref. 9 for the definition and convention).

These states can be easily identified in Fig. 3(a), which shows the spectrum for $J^{\prime} / J=0.2$. In particular, the degeneracies and the symmetry properties are in complete agreement with group-theoretical calculations. ${ }^{18}$ This structure is also present in the spectra obtained for all values of $J^{\prime} / J$ larger than 0.2 up to $J^{\prime}=J$, including $J^{\prime}=J / 2$, a special point for the 12-site cluster where the tower structure can be explicitly proven. ${ }^{18}$ By contrast, the spectrum for $J^{\prime}=0$ is shown in Fig. 3(b). Clearly, the tower structure is lost: The excitation energies are not aligned on a straight line, and the symmetry and degeneracy of the corresponding excited states do not agree with group theory. This behavior is consistent with a quantum phase transition between $J^{\prime} / J=0$ and 0.2. Due to the very rapid increase of the size of the Hilbert space, we could not study larger systems to check that the tower structure is still there for large enough $J^{\prime} / J$, and to perform a finite-size scaling of the slope. Still, the comparison of the spin-wave results, obtained in the thermodynamic limit, with these exact diagonalization results gives a very strong indication that there is a quantum phase transition between a four-sublattice ordered state for large enough $J^{\prime} / J$ and some kind of spin liquid for $J^{\prime}=0$. 

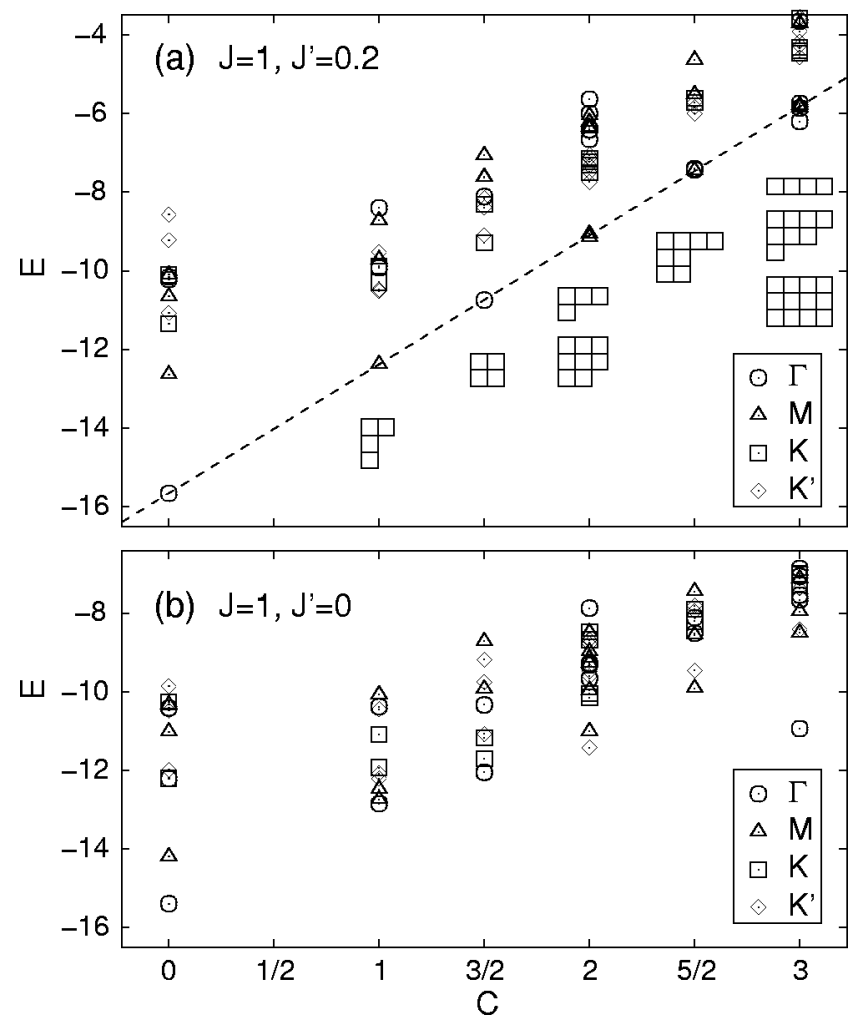

FIG. 3. Low-energy spectrum of the 12-site cluster as a function of the first Casimir $C$ for (a) $J^{\prime} / J=0.2$ and (b) $J^{\prime}=0$. The Anderson tower of states is clearly identified for $J^{\prime} / J=0.2$ (upper plot). The Young diagrams corresponding to the states that build up the tower are depicted.

What is the nature of this disordered ground state? For $\mathrm{SU}(2)$ models, experience with frustrated systems has shown that it is useful to look at the structure of the low-lying singlet spectrum of a finite system, which can contain a single state in valence-bond solids with no lattice-symmetry breaking, four states for true spin liquids on a torus (topological degeneracy $\left.{ }^{19}\right)$, a finite number of states in valencebond solids with lattice-symmetry breaking, or a proliferation of low-lying singlets, as in the spin-1/2 kagome antiferromagnet. ${ }^{20}$ In the present case, exact diagonalization results on finite-size clusters with 12 and 16 sites show that there are four (respectively, six) singlets before the first multiplet, but no clear tendency can be identified.

To reach larger system sizes, we have decided to resort to variational calculations in the spirit of the resonating valence-bond approach to frustrated SU(2) magnets. ${ }^{21}$ There are significant differences though. First, one needs at least four sites to make an SU(4) singlet. Now, among all possible four-site clusters, the particular role of the singlet plaquettes was pointed out by Li et al.: $:^{7}$ On a triangular lattice, each plaquette has a diagonal energy of - (13/4) J, which is much lower than the diagonal energy of the LRO state (which is 0 ). Thus the singlet plaquettes are a good starting point for a variational calculation. To implement the method, we define a Hilbert space which is spanned by the different coverings $\left|\psi_{j}\right\rangle\left(j=1, \ldots, N_{\text {cov }}\right)$ of the finite-size cluster with singlet plaquettes. The eigenenergies are then the solution of the generalized eigenvalue problem $\operatorname{det}\left(E O_{i j}-H_{i j}\right)=0$, where $O_{i j}=\left\langle\psi_{i} \mid \psi_{j}\right\rangle$ and $H_{i j}=\left\langle\psi_{i}|\mathcal{H}| \psi_{j}\right\rangle$. A systematic way to improve the method is to double the Hilbert space by including the states $\left|\psi_{j}^{\prime}\right\rangle=H\left|\psi_{j}\right\rangle$, which leads to the

$$
\left|\left[\begin{array}{cc}
H_{i j} & H_{i j}^{(2)} \\
H_{i j}^{(2)} & H_{i j}^{(3)}
\end{array}\right]-E\left[\begin{array}{cc}
O_{i j} & H_{i j} \\
H_{i j} & H_{i j}^{(2)}
\end{array}\right]\right|=0
$$

$2 N_{\text {cov }} \times 2 N_{\text {cov }}$ eigenvalue problem, where $H_{i j}^{(n)}$ $=\left\langle\psi_{i}\left|\mathcal{H}^{n}\right| \psi_{j}\right\rangle$. This step can be repeated to include matrix elements of further powers of $\mathcal{H}^{n}$. The efficiency of the method is illustrated in Table I.

The advantage of the variational approach is that we could perform calculations for much larger clusters, up to 36 sites with two additional steps, 48 sites with one additional step, and 64 sites with no additional step. For clarity, we focus the discussion on two natural possibilities: (i) The singlet plaquettes form a pattern with long-range order, the most natural candidate being then the static covering (plaquette array) as in the square lattice ${ }^{9}$ [see Fig. 4(a)]; (ii) the plaquettes undergo strong resonances between configurations that differ only locally, the smallest pattern that allows such a resonance being the diamond depicted in Fig. 4(b).

The first possibility is definitely not favored by our variational results: For both the 36-site and the 48-site clusters, the plaquette covering which has the largest weight in the ground-state wave function is not the square covering, but a

TABLE I. Energy, degeneracy, and wave vector of the low-lying singlet states of the 12- and 16-site symmetric clusters obtained for $J=1$ and $J^{\prime}=0$ by exact diagonalization and by the variational approach with 0 , one, and two additional steps. The second column is the energy of a single covering (no resonance between plaquettes). The number of plaquette coverings is equal to 36 (84) for 12 (16) sites, while there are 462 (24 024) singlets in the full Hilbert space. In the exact diagonalization spectrum, these states lie below the first multiplet ( $E=-12.841$ and -18.451 for 12 and 16 sites, respectively).

\begin{tabular}{cccccccc}
\hline \hline$N$ & Diagonal energy & 0 steps & one step & two steps & Exact & Degeneracy & $k$ \\
\hline 12 & -9.75 & -14.657 & -15.314 & -15.381 & -15.384 & 1 & $\Gamma$ \\
12 & -9.75 & -12.206 & -13.781 & -14.141 & -14.188 & 3 & $M$ \\
16 & -13 & -19.253 & -20.935 & -21.068 & -21.079 & 1 & $\Gamma$ \\
16 & -13 & -15.767 & -17.979 & -18.634 & -18.908 & 2 & $\Gamma$ \\
16 & -13 & -16.798 & -18.252 & -18.624 & -18.754 & 3 & $M$ \\
\hline \hline
\end{tabular}




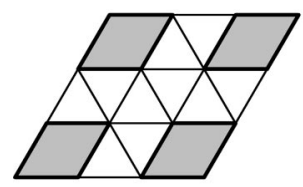

(a)

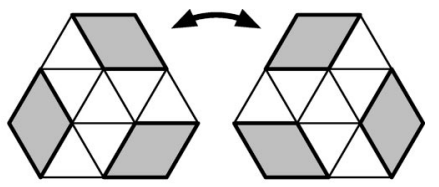

(b)
FIG. 4. Three remarkable covering patterns of the triangular lattice with plaquettes. The last two resonate strongly.

diamond covering (see Fig. 5). This fact is actually easy to understand: The matrix elements corresponding to the resonance of Fig. 4(b) dominate the secular equation, and they are completely suppressed in the square pattern of Fig. 4(a).

The effect of these resonances on the ground-state correlations is a delicate issue. The ground states of the 36-site and 48-site clusters are consistent with a long-range pattern for the diamond covering of the triangular lattice, but given the very small sizes from that point of view (the 36- and 48 -site clusters can accommodate three and four diamonds, respectively) this result is probably not significant. What is likely to be more relevant is the amplitude of the processes that would enter a description in terms of an effective Hamiltonian. The two plaquette coverings inside a diamond can be described by an Ising variable, and the resonance gives rise to a transverse field of order $J / 2$. The spectra obtained for the 36-site and 48-site clusters are then consistent with a very small potential-energy term with two- and three-body interactions. A detailed analysis of this model lies beyond the scope of the present paper, but given the prominence of the kinetic-energy term, it is very likely that the system is the equivalent of the disordered phase of the Ising model in a transverse field. ${ }^{22}$

In summary, this variational approach strongly suggests that the ground state is a plaquette liquid with no four-site plaquette long-range order, and with strong local resonances between the configurations of Fig. 4(b). The presence of long-range order associated to a specific pattern of diamond covering can neither be ascertained nor excluded on the basis

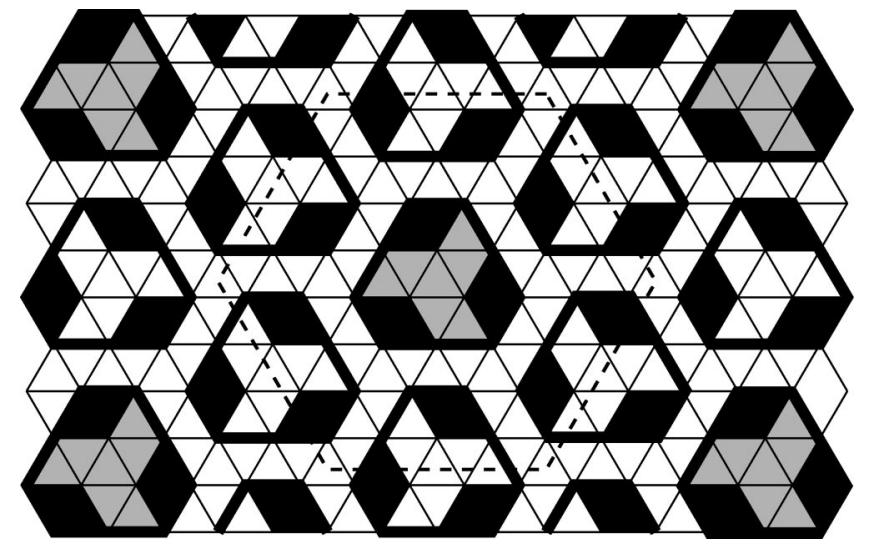

FIG. 5. Dominant plaquette covering in the ground state of the 48 -site cluster (dashed line). The diamonds making up the new superlattice are shaded using different grays for the two patterns of Fig. 4(b).

of the present results. We just note that, if no longer-range correlations are present, this plaquette liquid is expected, as is its $\mathrm{SU}(2)$ counterparts, to exhibit topological degeneracy. ${ }^{19}$

To conclude, we have shown that it is possible to order the SU(4) Heisenberg model for sufficiently large nextnearest-neighbor repulsion, without the need to introduce anisotropy, and we have identified a quantum phase transition around $J^{\prime} / J=0.12$. For $J^{\prime}=0$, which corresponds to a minimal model of $\mathrm{LiNiO}_{2}$, we have shown that the ground state is a spin and orbital liquid, and we have shown that simple objects such as dimers of plaquettes do not develop longrange order. These results are consistent with the absence of any kind of phase transition in $\mathrm{LiNiO}_{2}$. More generally, Mott insulators with orbital degeneracy and the appropriate geometry emerge as potential candidates for completely order-free spin liquids with topological degeneracy.

We acknowledge the financial support of the Hungarian OTKA Grant Nos. T038162 and D32689, Bolyai Grant No. 118/99, and the Swiss National Fund.
${ }^{1}$ L.-F. Feiner et al., Phys. Rev. Lett. 78, 2799 (1997).

${ }^{2}$ Y. Kitaoka et al., J. Phys. Soc. Jpn. 67, 3703 (1998); F. Reynaud et al., Phys. Rev. Lett. 86, 3638 (2001).

${ }^{3}$ B. Bernu et al., Phys. Rev. Lett. 69, 2590 (1992).

${ }^{4}$ H. Nakamura et al., Phys. Rev. Lett. 79, 3779 (1997); G. Mihály et al., Phys. Rev. B 61, R7831 (2000).

${ }^{5}$ K.I. Kugel and D.I. Khomskii, Usp. Fiz Nauk. 136, 621 (1982) [Sov. Phys. Usp. 25, 231 (1982)].

${ }^{6}$ B. Sutherland, Phys. Rev. B 12, 3795 (1975); Y. Yamashita et al., ibid. 58, 9114 (1998); B. Frischmuth et al., Phys. Rev. Lett. 82, 835 (1999).

${ }^{7}$ Y.Q. Li et al., Phys. Rev. Lett. 81, 3527 (1998).

${ }^{8}$ M. van den Bossche et al., Phys. Rev. Lett. 86, 4124 (2001).

${ }^{9}$ M. van den Bossche et al., Eur. Phys. J. B 17, 367 (2000).

${ }^{10}$ A. Mishra et al., Phys. Rev. B 65, 214411 (2002).

${ }^{11}$ S.Q. Shen, Phys. Rev. B 66, 214516 (2002).

${ }^{12}$ P. Thalmeier et al., J. Phys. Soc. Jpn. 67, 2363 (1998).

${ }^{13}$ D.P. Arovas and A. Auerbach, Phys. Rev. B 52, 10114 (1995); G.
Santoro et al., Phys. Rev. Lett. 83, 3065 (2001); K. Harada et al., ibid. 90, 117203 (2003).

${ }^{14}$ I. Affleck and J.B. Marston, Phys. Rev. B 37, 3774 (1988); N. Read and S. Sachdev, Phys. Rev. Lett. 62, 1694 (1989).

${ }^{15}$ A. Joshi et al., Phys. Rev. B 60, 6584 (1999); F.P. Onufrieva, Zh. Éksp. Teor. Fiz. 89, 2270 (1985) [Sov. Phys. JETP 62, 1311 (1985)].

${ }^{16}$ P.W. Anderson, Phys. Rev. 86, 694 (1952).

${ }^{17}$ P. Lecheminant et al., Phys. Rev. B 52, 6647 (1995).

${ }^{18}$ K. Penc, M. Mambrini, P. Fazekas, and F. Mila (unpublished).

${ }^{19}$ For a recent discussion see G. Misguich et al., Eur. Phys. J. B 26, 167 (2002), and references therein.

${ }^{20}$ P. Lecheminant et al., Phys. Rev. B 56, 2521 (1997); F. Mila, Phys. Rev. Lett. 81, 2356 (1998).

${ }^{21}$ P.W. Anderson, Mater. Res. Bull. 8, 153 (1973); P. Fazekas and P.W. Anderson, Philos. Mag. 30, 423 (1974).

${ }^{22}$ R. Moessner and S.L. Sondhi, Phys. Rev. B 63, 224401 (2001). 